\title{
Estilos cognitivos e personalidade: um estudo exploratório de evidências de validade
}

\author{
Acácia Aparecida Angeli dos Santos ${ }^{1}$ \\ Fermino Fernandes Sisto ${ }^{2}$ \\ Rosana Maria Mohallem Martins ${ }^{3}$
}

\begin{abstract}
Resumo
Com o objetivo de explorar evidências de validade para uma escala de estilos cognitivos em relação a traços de personalidade, foram estudados 135 universitários de ambos os sexos. Os dois instrumentos foram aplicados coletivamente em sala de aula. A escala para medir estilos cognitivos informava sobre os estilos convergente, divergente, holista, serialista, reflexividade e impulsividade; enquanto o inventário de adjetivos marcadores da personalidade fornecia dados sobre os traços realização, extroversão, neuroticismo, abertura e socialização. Com base em suas definições esperavam-se encontrar nove correlações entre os estilos cognitivos e os traços de personalidade. Os dados obtidos não mostraram diferenças nas dimensões de estilo cognitivo relacionadas ao gênero e à idade. Também foram encontradas 12 correlações significativas, cinco delas confirmando as hipóteses e as outras não. Embora algumas evidências de validade para a escala de estilos cognitivos tenham sido detectadas, já que algumas hipóteses foram confirmadas, sugere-se a realização de novas pesquisas que explorem mais esse construto, tanto teoricamente como em relação a validade.

Palavras-Chave: Avaliação psicológica; Validade; Traços de personalidade; Big-five; Estilos cognitivos; Estudantes universitários.
\end{abstract}

\section{Cognitive styles and personality: an exploratory study of validity evidences}

\begin{abstract}
135 undergraduates, of both genders, were studied to explore validity evidences of a scale of cognitive styles in relation to personality traits. The two tests were collectively applied in classroom. The scale of cognitive styles assessed about the convergent, divergent, holist, serialist, reflexivity and impulsiveness styles; whereas the personality inventory of adjectives markers assessed the agreeableness, extraversion, conscientiousness, neuroticism, openness. Based on their definitions, nine correlations were expected to find among cognitive styles and personality traits. The obtained data did not show differences in the dimensions of cognitive style related to gender and age. Besides, 12 significant correlations were found, five of them confirming the hypotheses, but not the other ones. Although some validity evidences for the scale of cognitive styles have been detected, since some hypothesis were confirmed, the accomplishment of new researches is suggested to explore more that construct, considering both the theory and evidences of validity.
\end{abstract}

Keywords: Psychological assessment; Validity; Personality traits; Big-five; Cognitive styles; Undergraduates.

\section{Introdução}

Acredita-se que haja diferenças entre as formas como as pessoas comumente pensam e sentem as situações com as quais se defrontam. Assim, haveria tendências diferenciadas nas formas de aprender e relacionar os dados da realidade e de elaborar conclusões sobre eles. Uma das formas para se estudar essas diferenças consubstanciou-se no construto estilo cognitivo e Lemes (1998) relata sua origem nos estudos sobre cognição humana dentro da perspectiva diferencial, referindo-se a Galton (1883), James (1890), Cattel (1890) e Barllet (1932), entre outros, como os seus primeiros estudiosos.

Autores, como Allport (1973), já se referiam à existência de disposições proceptivas ou disposição de resposta perceptual e conceitos pessoais $\mathrm{O}$ termo procepção engloba as relações passadas com o mundo, as disposições emocionais e expectativas para o futuro em relação à cultura em que vive, preservando a individualidade de cada um. Existir como pessoa e desenvolver a própria visão de mundo determinariam o estilo cognitivo.

Embora exista uma variedade de definições sobre estilos cognitivos e sejam definições distintas, Messick (1984) destaca que entre elas há elementos que

\footnotetext{
1 Universidade São Francisco.

2 Universidade São Francisco.

${ }^{3}$ Centro Universitário de Itajubá-MG.

E-mail: acaciasantos@terra.com.br
} 
se sobrepõem, sem torná-las mutuamente exclusivas. Considera que os estilos cognitivos refletem diferenças individuais na organização cognitiva da pessoa e os vê como elemento mediador entre a habilidade e a personalidade. Refletem o funcionamento metacognitivo dos indivíduos, organizando e controlando, tanto o processamento de informação como as respostas emocionais. Nesse sentido, parece razoável pressupor que os estilos servem para discriminar o limite entre cognição e personalidade, apesar de serem fruto da integração dos aspectos cognitivo e afetivo (motivações do funcionamento individual).

Para Bariani (1998) os estilos são estruturas relativamente estáveis e podem sofrer impacto de experiências vividas durante os anos de escolaridade, inclusive na etapa do ensino superior. Contudo, não se pode afirmar quais são os fatores mais marcantes, se as estruturas curriculares dos cursos, o ambiente acadêmico ou a convivência com outros grupos culturais. O estudo de Bariani, Escher, Santos e Pitta (2000), com universitários, permitiu verificar que a experiência em iniciação científica pôde ser um elemento modificador dos estilos dos estudantes. Os resultados obtidos indicaram uma tendência de acentuação dos estilos já existentes, sendo que alguns fatores contribuíram para isso, tais como a atuação do orientador, as características das atividades realizadas na iniciação científica e a situação de trabalho em grupo.

A preferência cognitiva do estudante é uma variável independente importante, pois contribui exclusivamente na realização e organização cognitiva no contexto de habilidade geral, organização cognitiva anterior e variáveis de conhecimento (McRobbie, 1991; Riding, 1997). Estudos realizados com universitários têm procurado explorar as características de estilo cognitivo com o desempenho acadêmico em áreas de conteúdo específico (Viswanathan, 1993; Armstrong, 2000; Zhang, 2002), bem como com outros aspectos, tais como a criatividade (Kaufman, 2002) e a inteligência (Riding \& Pearson, 1994; Sternberg, 1994; Riding \& Agrell, 1997).

No entanto, valendo-se de uma revisão sobre o assunto, Sternberg e Grigorenko (1997) concluíram que, nos últimos anos, as pesquisas sobre os estilos cognitivos diminuíram ante alguns resultados confusos sobre validade interna e externa do construto. A validade do construto estilo cognitivo é apoiada na evidência de que as dimensões são independentes, não se confundem com inteligência, mas interagem com os traços de personalidade.

Recentemente, Bariani, Sisto e Santos (2000) construíram um instrumento brasileiro, destinado à avaliação de estilos cognitivos, tendo sido encontradas evidências das validades de definição e de construto. A primeira foi obtida com base na classificação de juízes especialistas na área. As evidências de validade de construto apoiaram-se nos resultados da análise fatorial, a qual agrupou os itens em três fatores, com exclusão da dimensão dependência/independência de campo. Obteve-se a explicação de $36,8 \%$ da variância do fenômeno observado e a confirmação de que os estilos cognitivos são bipolares pela correlação inversa das três dimensões então obtidas, impulsividade/reflexividade de resposta, pensamento convergente/divergente e holista/serialista, descritos a seguir.

A dimensão impulsividade/reflexividade de resposta está ligada a um aspecto mais amplo, a organização da atenção. A impulsividade implica daremse respostas imediatas e freqüentemente imprecisas, com pouca ponderação e organização prévia. Já a reflexividade refere-se a uma tendência para analisar e diferenciar uma configuração complexa de estímulos. As pessoas com preponderância nesse estilo possuiriam pensamentos mais organizados e seqüenciados, utilizando uma estratégia mais analítica na realização das tarefas (Bariani, 1998, Bariani, Sisto \& Santos, 2000).

Há uma identificação do pensamento convergente com o pensamento lógico e o raciocínio. Indivíduos com essa dimensão acentuada são hábeis em lidar com problemas que requerem uma clara resposta convencional (uma solução correta), com base nas informações fornecidas. São inibidos emocionalmente e identificados como mais conformistas, disciplinados e conservadores. O pensamento divergente é associado à criatividade, a respostas imaginativas, originais $\mathrm{e}$ fluentes. Os indivíduos com essa predominância preferem problemas informais, sendo hábeis em tratar de problemas que demandam a generalização de várias respostas igualmente aceitáveis. Socialmente são considerados irritadiços, disruptivos e até ameaçadores (Bariani, 1998; Bariani, Sisto \& Santos, 2000)

Indivíduos com pensamento holista dão maior ênfase ao contexto global desde o início de uma tarefa. Preferem examinar uma grande quantidade de dados, buscando padrões e relações entre eles. Usam hipóteses mais complexas, às quais combinam diversos dados. Já, os serialistas dão maior ênfase a tópicos separados e em seqüências lógicas, buscando padrões e relações somente mais tarde no processo, para confirmar ou não suas hipóteses. Usam hipóteses mais simples e uma abordagem lógico-linear (Riding \& Wheeler, 1995; Santos, Bariani \& Cerqueira, 2000; Bariani, Sisto \& Santos, 2000; Zhang, 2002).

A literatura registra um grupo de pesquisas que procuraram explorar possíveis relações entre o estilo de pensar e aprender e traços de personalidade. Apóiam-se na hipótese de que o construto estilo cognitivo encontrase na zona limítrofe do construto personalidade (Rothstein, Paunonen, Rush, \& King, 1994; Riding, 
Burton, Rees \& Sharratt, 1995; Riding \& Wigley, 1997; entre outros).

Muitos estudiosos definiram personalidade com base em medidas de características específicas, que aparecem sob muitas denominações, tais como indiferença, animosidade, imprudência, brandura, retidão, entre outras. Se, por um lado, é importante destacar que a variedade de características de personalidade é grande; por outro, vale lembrar que existem muitos estudos apontando para as possíveis relações entre tais características/traços (Peabody \& Goldberg, 1989, por exemplo).

Há muito que a personalidade vem sendo estudada com base em procedimentos empíricos. Cattel (1945) tornou-se um dos pioneiros na construção de uma taxonomia dos termos descritivos desse construto. Em razão das correlações encontradas entre eles, dividiu-os em vários grupos e utilizou-os em estudos subseqüentes, alegando a existência de 12 fatores que os englobariam. Estudos posteriores, que incluíram também outros grupos de variáveis, têm apontado consistentemente para a estabilidade do modelo de apenas cinco fatores (Fiske, 1949; Norman, 1963; Borgatta, 1964; Digman \& Inouye, 1986; McCrae \& Costa, 1985 e 1987; Goldberg, 1990, 1993; Digman, 1997).

No Brasil, Hutz e colaboradores (1998) encontraram evidências de validação para o modelo Cinco Grandes Fatores (CGF). Análises fatoriais, utilizando diferentes métodos de extração, mostraram que uma solução de cinco fatores é apropriada e a estrutura dos itens se mantém estável. Os fatores extraídos são equivalentes aos descritos na literatura como modelo Big Five e se organizam como Fator I - socialização, Fator II - extroversão, Fator III - realização, Fator IV - neuroticismo e Fator V - abertura, descritos a seguir.

Socialização (Fator I). Refere-se aos tipos de interação, sendo uma dimensão interpessoal, que se estende da compaixão ao antagonismo. Pessoas com escores altos tendem a ser bondosas, generosas, afáveis, altruístas e prestativas. São ávidas para ajudar os outros e acreditam que a maioria das pessoas é assim. Por outro lado, as pessoas com baixo escore nesse fator tendem a ser cínicas, irritáveis, não cooperativas, manipuladoras e vingativas.

Extroversão (Fator II). Relaciona-se à quantidade e intensidade das interações interpessoais, nível de atividade, capacidade de alegrar-se e necessidade de estimulação. Pessoas com escore alto em extroversão tendem a ser ativas, otimistas, afetuosas, falantes e sociáveis; enquanto pessoas com escore baixo apresentam tendências à introversão, sendo reservadas, quietas, indiferentes e independentes.

Realização (Fator III). Representa o grau de persistência, controle, organização e motivação para alcançar objetivos. Escores altos neste fator indicam pessoas organizadas, decididas, confiáveis, pontuais, trabalhadoras, perseverantes, ambiciosas e escrupulosas. Em contrapartida, escores baixos indicam pessoas não confiáveis, preguiçosas, descuidadas e negligentes.

Neuroticismo (Fator IV). Está relacionado ao nível crônico de ajustamento emocional e com a instabilidade. Alto escore identifica indivíduos propensos ao sofrimento psicológico, com tendência à ansiedade, depressão, hostilidade, impulsividade, autocrítica, vulnerabilidade, baixa tolerância a frustrações e idéias não realistas.

Abertura (Fator V). Refere-se ao reconhecimento da importância de ter novas experiências e a comportamentos exploratórios. Pessoas com alto escore tendem a ser imaginativas, criativas, curiosas, divertemse com novas idéias e com valores não convencionais; enquanto pessoas com baixo escore tendem a ser convencionais nas suas crenças e atitudes, conservadoras nas suas preferências, rígidas em suas crenças, dogmáticas e menos responsivas emocionalmente.

Pesquisas têm sido desenvolvidas, buscando relacionar os fatores de personalidade como preditores de desempenho em seleção de pessoal para diversas carreiras. A revisão de literatura baseada no modelo de cinco fatores tem indicado a existência de relações com uma variedade de critérios de desempenho profissional (Hough, Eaton, Dunette, Kamp \& McCloy, 1990; Barrick \& Mount, 1991; Tett, Jackson \& Rothstein, 1991). No entanto, outros estudos não têm encontrado a mesma consistência do modelo de cinco fatores na predição do comportamento, o que pode estar relacionado ao tipo de instrumento utilizado, ou seja, à utilização de versões com maior ou menor número de itens. Independentemente disso, as conclusões reforçam a validade do instrumento, mas apontam para a necessidade de novos estudos e cautela na sua utilização, considerando a possibilidade de erro ou de pouca acuidade para prever alguns tipos de comportamentos (Paunonen, 1998; Paunonen \& Ashton, 2001).

Uma vez que o instrumento de avaliação de estilos cognitivos, aqui focalizado, apresenta evidências de validação de definição e de construto, considerou-se pertinente buscar-se outros tipos de evidências de validade. A sua comparação com um instrumento que mede traços de personalidade foi considerada como uma alternativa de se encontrar evidências de validade concorrente para o instrumento, já que a literatura acusa relações entre essas variáveis (Riding \& Wigley, 1997; Rothstein, Paunonen, Rush, \& King, 1994; Sternberg e Grigorenko (1997); entre outros). Nesse contexto, algumas hipóteses foram levantadas para este estudo, que tem como participantes sujeitos em fase de formação profissional. 


\section{Hipóteses}

O estilo cognitivo convergente se refere a pessoas geralmente inibidas emocionalmente e identificadas como mais conformistas, disciplinadas e conservadoras. Em razão dessas características esperam-se correlações com dois traços de personalidade. Com o traço de personalidade realização existe a expectativa de correlação positiva e significativa, pois alta pontuação nesse fator indicaria pessoas caracterizadas como organizadas, decididas e perseverantes. Há também a expectativa de correlação negativa e significativa com o traço de extroversão, pois baixas pontuações indicariam pessoas reservadas, quietas, independentes e indiferentes.

O estilo cognitivo reflexividade englobaria pessoas possuidoras de formas de pensar organizadas e seqüenciadas e com tendência à ponderação prévia para suas respostas. Aventa-se a possibilidade de correlação positiva e significativa com o traço de personalidade realização, pois alta pontuação nesse fator indicaria pessoas caracterizadas como organizadas, decididas e perseverantes; como também de correlação negativa com neuroticismo, pois esse traço se refere a pessoas com instabilidade emocional e alta pontuação indicaria pessoas com tendência à ansiedade, depressão, impulsividade, vulnerabilidade, baixa tolerância a frustrações e idéias não realistas, entre outras.

Pessoas com características do estilo cognitivo impulsividade se deteriam pouco em ponderação e organização prévias para dar uma resposta. Em razão dessas tendências esperam-se correlações com dois traços de personalidade. Por um lado, uma correlação positiva com neuroticismo, principalmente em razão da instabilidade emocional e pelos sintomas de ansiedade, hostilidade, impulsividade, vulnerabilidade e idéias não realistas, peculiaridades de uma pessoa com tendência ao neuroticismo e, por outro, correlação também positiva com o traço de extroversão porque pessoas com alta pontuação tendem a ser ativas, otimistas, afetuosas, falantes e sociáveis.

No que tange ao estilo cognitivo divergente, a conduta das pessoas estaria associada à criatividade, a respostas imaginativas, originais e fluentes. As pessoas com esse tipo de estilo preferem problemas informais, são hábeis em tratar de problemas que demandam várias respostas igualmente aceitáveis. A expectativa é que se correlacione positivamente com o traço de personalidade abertura, pois pessoas com esse traço dão importância a novas experiências e a comportamentos exploratórios, tendem a ser imaginativas, criativas, curiosas e divertemse com novas idéias e valores não convencionais.

Além disso, não se espera qualquer correlação significativa com os estilos cognitivos holista e serialista, porque suas descrições se referem muito mais a uma forma de abordagem, sem muitas indicações do tipo de pessoa. Nessa mesma direção não se aventa a possibilidade do traço de personalidade socialização se correlacionar com qualquer estilo cognitivo.

\section{Método}

\section{Participantes}

Foram estudados 135 universitários, 58 do gênero masculino e 77 do gênero feminino. Todos eles eram calouros do segundo semestre, provenientes dos cursos de administração $(\mathrm{n}=39)$, psicologia $(\mathrm{n}=29)$, computação $(\mathrm{n}=24)$ e farmácia ( $\mathrm{n}=43)$. A idade mínima foi de 17 e a máxima de 47, com uma média de 22 anos e desvio padrão de 5,18.

\section{Instrumentos}

Foram utilizados dois instrumentos. O primeiro a ser descrito destinou-se à avaliação dos estilos cognitivos e o segundo à avaliação da personalidade.

Escala de Avaliação de Estilos Cognitivos

É uma escala do tipo Likert desenvolvida por Bariani, Sisto e Santos (2000) para avaliar as dimensões holista/serialista, impulsividade/reflexividade e pensamento divergente/convergente, com seis itens para cada dimensão. As pessoas devem responder indicando o grau de concordância e discordância em relação aos dezoito itens que compõem a escala, apresentados em seqüência aleatória e independentemente do estilo que medem. Como resposta, o sujeito deve marcar com um "X" uma das categorias: DT (discordo totalmente), D (discordo), I (indeciso), C (concordo), CT (concordo totalmente), nos diferentes itens. É atribuído de 1 a 5 pontos a cada resposta, correspondendo respectivamente às categorias antes apresentadas. A pontuação de cada pessoas foi calculada pela soma dos pontos obtidos em cada estilo, separadamente.

\section{Big Five - Adjetivos Marcadores da Personalidade}

Este instrumento foi construído por Hutz e colaboradores (1998), destinado a avaliar a personalidade no contexto do modelo dos Cinco Grandes Fatores (CGF). Esse modelo é uma versão moderna da Teoria do Traço, que representa dimensões humanas básicas de forma consistente e replicável. A escala utilizada foi a versão simplificada, composta de 64 adjetivos. O sujeito deve responder em que grau o adjetivo descreve características de sua personalidade. É uma escala Likert e apresenta cinco categorias de respostas, que variam de 1 a 5 pontos: (1) discordo totalmente; (2) discordo em parte; (3) nem concordo, nem discordo; (4) concordo em parte; (5) concordo totalmente. Os 64 itens são apresentados em uma seqüência aleatória, independentes do fator que os descreve, porém para cada fator há um número específico de adjetivos. O Fator I - socialização, 
apresenta 16 adjetivos; o Fator II - extroversão, apresenta 10; o Fator III - realização, apresenta 14; o Fator IV neuroticismo, apresenta 12 e o Fator V - abertura, apresenta 12 adjetivos. A pontuação final é obtida pela soma dos pontos em cada fator, porém, para os itens de pólos negativos, calcula-se $(6-\mathrm{R})$, sendo que o R se refere aos pontos correspondentes à resposta do sujeito.

\section{Procedimento}

Os sujeitos foram convidados a participar do estudo, sendo garantido o sigilo e a confidencialidade dos resultados. Foi explicado o objetivo do estudo e os que aceitaram assinaram um Termo de Consentimento. A aplicação dos instrumentos foi realizada coletivamente, em duas sessões e sem tempo determinado.

\section{Resultados}

Em relação aos seis estilos cognitivos, foram calculadas as médias, desvios padrões, apresentados na Tabela 1. Também são fornecidas as pontuações máximas e mínimas.

Tabela 1 - Estatística Descritiva das Dimensões dos Estilos Cognitivos

\begin{tabular}{lcccc}
\hline & Média & Desvio padrão & Pontuação mínima & Pontuação máxima \\
\hline Convergente & 8,14 & 2,143 & 3 & 14 \\
Divergente & 10,78 & 2,084 & 5 & 15 \\
Holista & 9,63 & 2,261 & 3 & 15 \\
Serialista & 10,04 & 1,934 & 4 & 15 \\
Impulsividade & 8,80 & 2,476 & 3 & 15 \\
Reflexividade & 10,59 & 2,480 & 3 & 15 \\
\hline
\end{tabular}

Para verificar se a intensidade dos estilos cognitivos se diferenciava por gênero, foi usada a prova $\mathrm{t}$ de Student. Em nenhuma das medidas as diferenças de médias foi considerada significativa em nível de 0,05 , sendo atribuídas ao acaso. Assim, os diferentes gêneros apresentaram intensidades semelhantes dos distintos estilos. Em relação às idades, tampouco foram encontradas correlações significativas no nível estipulado anteriormente.

Em relação aos traços de personalidade, também foram calculadas as médias, desvios padrões, apresentados na Tabela 2. Da mesma forma, são fornecidas as pontuações máximas e mínimas encontradas.

Tabela 2 - Estatística Descritiva dos Traços de Personalidade

\begin{tabular}{lcccc}
\hline & Média & Desvio padrão & Pontuação mínima & Pontuação máxima \\
\hline Socialização & 65,96 & 9,018 & 30 & 80 \\
Extroversão & 30,78 & 8,535 & 14 & 50 \\
Realização & 54,65 & 7,123 & 28 & 70 \\
Neuroticismo & 28,64 & 6,442 & 16 & 46 \\
Abertura & 42,83 & 6,477 & 21 & 56 \\
\hline
\end{tabular}

Para verificar se os gêneros apresentaram diferenças em relação aos traços de personalidade, foi usada a prova t de Student. Apenas um dos resultados encontrados foi estatisticamente significativo no nível de 0,05 , sugerindo que os diferentes gêneros apresentaram intensidades diferentes no que concerne ao traço socialização ( $\mathrm{t}=-2,007 \mathrm{e} \mathrm{p}=0,047)$ e que o gênero feminino teve pontuações mais elevadas (média de 67,29 e desvio padrão de 8,51) que o masculino (média de 64,14 e desvio padrão de 9,449). Por sua vez, as idades não apresentaram correlações significativas com as pontuações dos traços de personalidade, da mesma forma que no caso dos estilos cognitivos.

Estudo correlacional entre os estilos cognitivos e os traços de personalidade.

Os coeficientes de correlação entre os traços de personalidade e os estilos cognitivos, assim como os valores de $\mathrm{p}$, são apresentados na Tabela 3. De maneira geral, esses resultados sugerem que as hipóteses levantadas não foram corroboradas em sua totalidade. 
$\underline{\text { Tabela } 3 \text { - Coeficientes de Correlação e Valores de p entre os Estilos Cognitivos e os Traços de Personalidade (n=135) }}$

\begin{tabular}{lccccccc}
\hline & & Convergente & Divergente & Impulsividade & Reflexividade & Holista & Serialista \\
\hline Socialização & $\mathrm{r}$ & $-0,189$ & 0,259 & $-0,092$ & 0,005 & 0,002 & 0,071 \\
& $\mathrm{p}$ & 0,029 & 0,003 & 0,293 & 0,956 & 0,983 & 0,420 \\
Extroversão & $\mathrm{r}$ & $-0,301$ & 0,288 & $-0,134$ & 0,160 & $-0,102$ & 0,010 \\
& $\mathrm{p}$ & 0,000 & 0,001 & 0,123 & 0,063 & 0,240 & 0,913 \\
\multirow{2}{*}{ Realização } & $\mathrm{r}$ & $-0,123$ & 0,203 & $-0,362$ & 0,317 & $-0,107$ & 0,131 \\
& $\mathrm{p}$ & 0,159 & 0,019 & 0,000 & 0,000 & 0,219 & 0,135 \\
\multirow{2}{*}{ Neuroticismo } & $\mathrm{r}$ & 0,100 & $-0,261$ & 0,118 & $-0,204$ & 0,229 & $-0,058$ \\
& $\mathrm{p}$ & 0,253 & 0,002 & 0,176 & 0,017 & 0,008 & 0,508 \\
Abertura & $\mathrm{r}$ & $-0,105$ & 0,472 & $-0,044$ & 0,191 & $-0,069$ & 0,067 \\
& $\mathrm{p}$ & 0,231 & 0,000 & 0,615 & 0,026 & 0,425 & 0,443 \\
\hline
\end{tabular}

A hipótese levantada em relação ao estilo cognitivo convergente afirmava uma correlação positiva com o traço de personalidade realização e negativa com extroversão. No que tange à correlação negativa com extroversão, foi confirmada, pois significativa, apesar de baixa. No entanto, os dados encontrados não confirmaram a sua relação com o traço de personalidade realização, já que a correlação, além de não ser significativa, foi negativa. No entanto, foi registrada uma correlação negativa significativa com o traço socialização.

Para o estilo cognitivo divergente, a hipótese previa uma correlação positiva com o traço de personalidade abertura. Os resultados encontrados acusaram correlação significativa com todos os traços de personalidade, um indicativo de não-discriminação. No entanto, há que se ressaltar que a correlação encontrada com o traço de personalidade abertura foi a mais alta $(\mathrm{r}=0,472)$.

No que concerne ao estilo cognitivo impulsividade, esperava-se uma correlação positiva com o traço de personalidade neuroticismo e extroversão e negativa com realização. Entretanto, apenas a correlação negativa com o traço de personalidade realização se confirmou.

A hipótese referente ao estilo cognitivo reflexividade indicava uma correlação positiva com o traço de personalidade realização e negativa com os traços extroversão e neuroticismo. Foram confirmadas as relações com os traços neuroticismo e realização e não com a extroversão. Além disso, foi encontrada uma correlação positiva e significativa com o traço abertura, não prevista inicialmente.

Não se esperava correlação entre os estilos holista e serialista com os traços de personalidade estudados, nem o traço de personalidade socialização estaria relacionado a algum estilo cognitivo. No entanto, o estilo cognitivo holista apresentou correlação positiva e significativa com o traço de personalidade neuroticismo, enquanto o traço de personalidade socialização mostrou correlação negativa e significativa com o estilo cognitivo convergente e positiva e significativa com o estilo divergente. Nesse grupo, apenas o estilo cognitivo serialista confirmou a hipótese de não apresentar correlação com os traços de personalidade em estudo.

Conforme as hipóteses levantadas, procedeu-se à interpretação dos resultados, considerando-se aquelas que puderam ser confirmadas ou não, com base nos dados obtidos com a amostra estudada.

\section{Discussão}

Os dados aqui apresentados não apontaram para diferenças nas dimensões de estilo cognitivo relacionadas ao gênero e à idade, o que foi congruente com os resultados obtidos, respectivamente, por Riding e colaboradores (1995) e Riding e Wheeler (1995) ao analisarem algumas dimensões do estilo cognitivo similares às aqui focalizadas. A única diferença encontrada na comparação entre os gêneros foi detectada no traço socialização, cuja pontuação é mais alta nas mulheres. A pesquisa realizada por Rothstein e colaboradores (1994) não detectou diferenças significativas entre os gêneros, mas os autores ressaltaram que os subgrupos eram numericamente muito diferentes (357 homens e 93 mulheres) e sugeriram que esse é um aspecto a ser mais bem explorado em outros estudos.

Com relação às hipóteses levantadas, eram esperadas nove correlações entre os estilos cognitivos convergente, divergente, reflexividade e impulsividade e os traços de personalidade realização, extroversão, neuroticismo e abertura. Não havia expectativa de correlações em relação aos estilos cognitivos holista e serialista nem em relação ao traço de personalidade socialização.

No entanto, foram encontradas 12 correlações significativas, cinco delas confirmando as hipóteses e as outras não aventadas como possíveis. Duas delas foram fornecidas por variáveis das quais não se esperava correlação, pois das três expectativas de correlação não significativa, apenas uma se confirmou, qual seja, o 
estilo cognitivo serialista. Uma análise de cada uma é apresentada a seguir.

O estilo cognitivo convergente refere-se a pessoas com predileção para o pensamento lógico, apresentando habilidade para lidar com problemas que requerem uma solução correta e preferência por problemas formais e tarefas estruturadas. Geralmente são inibidas emocionalmente e identificadas como mais conformistas, disciplinadas e conservadoras. Nesse contexto, havia a expectativa de correlação positiva e significativa com o traço de personalidade realização, pois alta pontuação nesse fator indicaria pessoas caracterizadas como organizadas, decididas e perseverantes, o que não foi confirmado. Havia também a expectativa de correlação negativa e significativa com o traço de extroversão, pois baixas pontuações indicariam pessoas reservadas, quietas, independentes e indiferentes, o que foi confirmado, já que a correlação negativa indica que conforme aumentou a pontuação em pensamento convergente, diminuiu a pontuação em extroversão. Houve também a correlação negativa significativa com o traço socialização, que indicaria que a pessoa com estilo cognitivo convergente teria como característica ser cínica, irritável, não cooperativa, manipuladora e vingativa. De fato, sem querer negar a realidade dos resultados nesse grupo pesquisado, o inesperado desses achados levanta a possibilidade de novas pesquisas para compreender um pouco mais as características psicológicas das pessoas com estilo convergente.

$\mathrm{O}$ estilo cognitivo reflexividade englobaria pessoas com melhor desempenho em tarefas que requerem processos detalhados e são possuidoras de formas de pensar organizadas e seqüenciadas e com tendência à ponderação prévia para suas respostas. Valendo-se dessas características, havia a expectativa de correlação positiva e significativa com o traço de personalidade realização, pois uma alta pontuação nesse fator indicaria pessoas caracterizadas como organizadas, decididas e perseverantes, o que foi confirmado, pois a correlação foi positiva e significativa. Levantou-se como hipótese, também, que haveria correlação negativa com neuroticismo, pois esse traço se refere a pessoas com problemas de ajustamento emocional e instabilidade e alta pontuação indicaria pessoas com tendência à ansiedade, depressão, hostilidade, impulsividade, autocrítica, vulnerabilidade, baixa tolerância a frustrações e idéias não realistas, o que também foi confirmado. Não foi confirmada, entretanto, correlação negativa com o traço extroversão, cuja correlação indicaria serem essas pessoas reservadas, quietas, indiferentes e independentes. No entanto, ocorreu correlação positiva com o traço abertura, que não era esperada. Esse dado indicaria que essas pessoas dariam importância a novas experiências e a comportamentos exploratórios, pois alta pontuação estaria relacionada a pessoas serem imaginativas, criativas, curiosas, além de se divertirem com novas idéias e com valores não convencionais. De todas as maneiras, esse acréscimo não parece contraditório e poderia indicar facetas congruentes da personalidade das pessoas com estilo cognitivo reflexividade.

As pessoas com características marcantes do estilo cognitivo impulsividade apresentariam um melhor desempenho em tarefas que requerem processos globais e para dar uma resposta se deteriam pouco em ponderação e organização prévias. Em razão dessas tendências esperava-se uma correlação positiva com neuroticismo, principalmente em razão da instabilidade emocional e pelos sintomas de ansiedade, depressão, hostilidade, impulsividade, autocrítica, vulnerabilidade, baixa tolerância à frustrações e idéias não realistas, que descrevem uma pessoa com tendência ao neuroticismo. Essa expectativa não foi confirmada. Esperava-se também correlação positiva com o traço de extroversão porque pessoas com alta pontuação tendem a ser ativas, otimistas, afetuosas, falantes e sociáveis, hipótese essa também não confirmada. No entanto, houve uma correlação negativa com o traço realização, que não estava incluída nas expectativas iniciais do estudo. Esse dado indicaria que as pessoas com alta pontuação no estilo impulsividade teriam baixas pontuações no traço realização e teriam como características ser preguiçosas, descuidadas, negligentes e não confiáveis. De certa forma não deixa de ser interessante essa informação, pois não parece se contrapor ao estilo cognitivo impulsividade.

Em se tratando do estilo cognitivo divergente, a conduta das pessoas estaria associada à criatividade, a respostas imaginativas, originais e fluentes. As pessoas com esse tipo de estilo preferem problemas informais, são hábeis em tratar de problemas que demandam várias respostas igualmente aceitáveis, e socialmente são consideradas irritadiças, disruptivas e até ameaçadoras. A expectativa era que se correlacionasse positivamente com o traço de personalidade abertura, pois pessoas com esse traço dão importância a novas experiências e a comportamentos exploratórios, tendem a ser imaginativas, criativas, curiosas e divertem-se com novas idéias e valores não convencionais. De fato, houve uma alta correlação entre essas duas medidas. No entanto, houve também correlação com todas as outras medidas de traços de personalidade, o que dificulta em muito uma interpretação coerente desse estilo à luz dos traços de personalidade.

Não se esperava nenhuma correlação com o estilo cognitivo holista, porque sua descrição se refere muito mais a uma forma de abordagem, sem muitas indicações do tipo de pessoa. Refere-se a dar ênfase ao contexto global desde o início de uma tarefa; a examinar uma grande quantidade de dados, buscando padrões e relações entre eles; e a lançar mão de hipóteses mais 
complexas para combinar diversos dados. No entanto, encontrou-se uma correlação positiva com neuroticismo, sugerindo que as pessoas com esse estilo tendem a ser instáveis e com problemas de ajustamento emocional, além de apresentarem tendência à ansiedade, depressão, hostilidade, impulsividade, autocrítica, vulnerabilidade, baixa tolerância à frustrações e idéias não realistas. De fato esse resultado não apresenta contradição, mas acrescenta uma informação interessante de ser pesquisada mais detidamente.

Não havia indicações para fazer suposições com respeito ao estilo cognitivo serialista e os resultados tampouco forneceram qualquer indicação nessa direção. Diferentemente do traço de personalidade socialização, que não se esperava correlacionar com qualquer estilo e que acabou mostrando correlações significativas com os estilos convergente e divergente.

Os achados neste estudo de certa forma sustentam as conclusões do estudo de revisão bibliográfica de Sternberg e Grigorenko (1997) no que se refere aos estilos cognitivos interagirem com os traços de personalidade. No entanto, é difícil sustentar integralmente que o construto estilo cognitivo encontra-se na zona limítrofe do construto personalidade tal como propuseram Riding e Wigley (1997) e Rothstein, Paunonen, Rush, e King (1994), entre outros, pois as correlações encontradas não foram altas o suficiente para tal sustentação nem ocorreram em todas as situações possíveis.

Não se pode deixar de ressaltar o fato de que o estilo serialista não se correlacionou com nenhum dos traços avaliados, o que era esperado, nem a literatura descreve com detalhes suficientes para se caracterizar seu perfil. O mesmo ocorre com o estilo holista em relação a dados para caracterizar seu perfil quanto a personalidade, o que, no entanto, não impossibilitou encontrar informações não contraditórias, aparentemente, em termos de traços de personalidade.

De fato, novas pesquisas devem ser realizadas para explorar um pouco mais essas relações. Ressalta-se principalmente o caso do estilo de pensamento divergente, que se correlacionou com todos os traços de personalidade, indicativo de que outros estudos devem ser feitos para interpretar esse dado, tanto teoricamente como em relação a validade. No entanto, algumas evidências de validade para a escala de estilos cognitivos ficaram transparentes, já que expectativas de relação foram confirmadas.

\section{Referências}

Allport, G. W. (1973). Personalidade: padrões e desenvolvimento. São Paulo: EPU, Editora da Universidade de São Paulo.

Armstrong, S. J. (2000) The influence of individual cognitive style on performance in management education. Educational Psychology, 20 (3), 323-339.
Bariani, I. C. D., Escher, C. A., Santos, L. A. D. \& Pitta, K. B. (2000). Estilos cognitivos de estudantes de psicologia: impacto da experiência em iniciação científica. Psicologia Escolar e Educacional, 4 (2), 41-49.

Bariani, I. C. D., Sisto, F. F., Santos, A. A. A. (2000). Construção de um instrumento de avaliação de estilos cognitivos. Em F. F. Sisto, E. T. B. Sbardelini \& R. Primi (Orgs.). Contextos e questões da avaliação psicológica. São Paulo: Casa do Psicólogo.

Bariani, I. C. D. (1998). Estilos cognitivos de universitários e iniciação científica. (Tese de Doutorado). Campinas: Universidade Estadual de Campinas - Faculdade de Educação.

Barrick, M. R. \& Mount, M. K. (1991). The Big Five personality dimensions and job performance: a metaanalysis. Personnel Psychology, 44, 1-26.

Borgatta, E. F. (1964). The structure of personality characteristics. Behavioral Science, 12, 8-17.

Cattell, R. B. (1945). The description of personality: principles and findings in a factor analysis. American Journal of Psychology, 58, 69-90.

Digman, J. M. (1997). Higher-order factors of the Big Five. Journal of Personality and Social Psychology, 73 (6), 1246-1256.

Digman, J. M. \& Inouye, J. (1986). Further specification of the five robust factors of personality. Journal of Personality and Social Psychology, 50, 116-123.

Fiske, D. W. (1949). Consistency of the factorial structures of personality ratings from different sources. Journal of Abnormal and Social Psycbology, 44, 329-344.

Goldberg, L. R. (1990). An alternative "Description of Personality" the big-five factor structure. Journal of Personality and Social Psychology, 59 (6), 1216-1229.

Goldberg, L. R. (1993) The structure of phenotypic personality traits. American Psychologist, 48 (1), 26-34.

Hough, L. M., Eaton, N. K., Dunnette, M. D., Kamp, J. D. \& McCloy, R. A. (1990). Criterion-related validities of personality constructs and the effect of response distortion on those validities. Journal of Applied Psychology, 75, 581-595.

Hutz, C. S., Nunes, C. H., Silveira, A. D., Serra, J., Antón, M. \& Wieczonek, L. S. (1998). O desenvolvimento de marcadores para a avaliação da personalidade no modelo dos cinco grandes fatores. Psicologia: Reflexão e Crítica, 11 (2), 395-410.

Kaufman, J. C. (2002). Narrative and paradigmatic thinking styles in creative writing and journalism students. Journal of Creative Behavior, 36 (3), 201-220.

Lemes, S. S. (1998). Os estilos cognitivos - dependência e 
independência de campo - na formação e no desempenho acadêmico em duas diferentes áreas de conhecimento: exatas e humanas. (Tese de Doutorado). São Paulo: Universidade de São Paulo - Instituto de Psicologia.

McCrae, R. R. \& Costa, P. T. (1985). Updating Norman's "adequate taxonomy": intelligence and personality dimensions in natural language and in questionnaires. Journal of Personality and Social Psychology, 49, 710-721.

McCrae, R. R. \& Costa, P. T. (1987). Validation of the five-factor model of personality across instruments and observers. Journal of Personality and Social Psychology, 52, 81-90.

McRobbie, C. J. (1991). Cognitive styles and cognitive structure. Science Education, 75 (2), 231-242.

Messick, S. (1984). The nature of cognitive style: Problems and promise in educational practice. Educational Psychologist, 19 (2), 59-74.

Norman, W. T. (1963). Toward an adequate taxonomy of personality attributes: replicated factor structure in peer nomination personality ratings. Journal of Abnormal and Social Psychology, 66, 574-583.

Paunonen, S. V. (1998). Hierarchical organization of personality and prediction of behavior. Journal of Personality and Social Psychology, 74, 538-556.

Paunonen S. V. \& Ashton M. C. (2001). Big Five factors and facets and the prediction of behavior. Journal of Personality and Social Psychology, 81 (3), 524-539.

Peabody, D. \& Goldberg, L. R. (1989). Some determinants of factor structures from personality-trait descriptors. Journal of Personality and Social Psychology, 57, 552-567.

Riding, R. J. (1997). On the nature of cognitive style. Educational Psychology, 17 (1/2), 29-49.

Riding, R. J. \& Pearson, F. (1994). The relationship between cognitive style and intelligence. EducationalPsychology, 14 (4), 413-425.

Riding, R. J. \& Wheeler, H. (1995). Occupational stress

Sobre os autores:

Acácia Aparecida Angeli dos Santos é psicóloga, doutora em Psicologia Escolar e Desenvolvimento Humano pelo Instituto de Psicologia da USP, professora da graduação em Psicologia e do Programa de Estudos PósGraduados em Psicologia da Universidade São Francisco- Itatiba-SP.

Fermino Fernandes Sisto é pedagogo, doutor em Educação pela Universidad Complutense de Madrid, livredocente pela UNICAMP, professor titular aposentado do Departamento de Psicologia Educacional da FEUNICAMP, professor da graduação em Psicologia e do Programa de Estudos Pós-Graduados em Psicologia da Universidade São Francisco - Itatiba-SP.

Rosana Maria Mohallem Martins é psicóloga, mestranda do Programa de Estudos Pós-Graduados em Psicologia da Universidade São Francisco - Itatiba-SP; professora da graduação em Psicologia no Centro Universitário de Itajubá-MG. and cognitive style in nurses. British Journal of Nursing, 4, 160-168.

Riding, R. J. \& Agrell, T. (1997). The effect of cognitve style and cognitive skills on school subject performance. Educational Studies, 23 (2), 311-323.

Riding, R. J. \& Wigley, S. (1997). The relationship between cognitive style and personality in further education students. Personality and Individual Differences, 23 (3), 379-389.

Riding, R. J., Burton, D., Rees, G. \& Sharratt, M. (1995). Cognitive style and personality in 12-year-old children. British Journal of Educational Psychology, 65 (1), 113-124.

Rothstein, M. G., Paunonen, S. V., Rush, J. C. \& King, G. A. (1994). Personality and cognitive ability predictors of performance in graduate business school. Journal of Educational Psychology, 86 (4), 516-530.

Santos, A. A. A., Bariani, I. C. D. \& Cerqueira, T. C. S. (2000). Estilos cognitivos e estilos de aprendizagem. psicologia para formação de professores. Petrópolis, RJ: Vozes; Bragança Paulista, SP: Universidade São Francisco.

Sternberg, R. J. (1994). Allowing for thinking styles. Educational Leadership, 52 (3), 36-40.

Sternberg, R. J. \& Grigorenko, E. L. (1997). Are cognitive styles still in style? American psychologist, 52 (7), 700-712.

Tett, R. P., Jackson, D. N. \& Rothstein, M. G. (1991). Personality measures as predictors of job performance: a meta-analytic review. Personnel Psychology, 44, 703-742.

Viswanathan, M. (1993). Measurement of individual differences in preference for numerical information. Journal of Applied Psychology, 78 (5), 741-752.

Zhang, L. (2002). Thinking styles: their relationships with modes of thinking and academic performance. Educational Psychology, 22 (3), 331-348.

Reformulado em junho de 2003 Aprovado em junbo de 2003 Em F. F. Sisto, G. C. Oliveira, L. D. T. Fini. Leituras de

Recebido em abril de 2003 
